\title{
Drone Flight Planning for Safe Urban Operations: UTM Requirements and Tools
}

\author{
Juan A. Besada, Ivan Campaña, Luca Bergesio, Ana M. Bernardos, Gonzalo de Miguel
}

\begin{abstract}
This paper describes the requirements of a flight planning tool for safe urban operations, which may be used to collaboratively design flights considering traffic constraints and limitations according to an unmanned traffic management system. Representative examples of flight planning are described, as calculated by a prototype flight planning tool.
\end{abstract}

Keywords - drone, fleet management, emergency management, unmanned traffic management system

\section{INTRODUCTION}

According to the European Drones Outlook Study [1], the market of civilian drone applications will be growing extremely fast in the next thirty years. Drone applications can be found in many different articles [2], and quite a few of them are focused on operations to be finally implemented in urban environments. It is easy to find examples of exploitation of drones for different purposes in the literature. A specially demanding application is that of e-commerce and delivery, where there are several proposals of the big technology companies, such as the Prime Air service of Amazon [3]. Also, air taxies may become a near future application [4]. In other cases, drone-based systems are used to enhance functionalities such as surveillance and reconnaissance, monitoring, mapping and photogrammetry, automatic fault detection or inventory tasks. There are also many examples urban infrastructure inspection scenarios in which drones are starting to be applied [5], [6], such as roofs inspection [7], real estate monitoring [8], construction management [9], etc. Additionally, monitoring of environmental gases for hazard assessment or air pollution management may complement in the city though UAVs. For example, authors in [10] describe the design of a Gas Sensing System ready to be mounted on any UAV. For city management, road inspection may be clearly enhanced using drones help to detect early signs of erosion and pavement distress [11], [12], [13], [14]. Similar approaches might be used for railway inspection [15].

The mission type obviously determines the type of flight to be completed [16], with clear differences for local (Visual Line of Sight VLOS) and remote (Beyond Visual Line of Sight, BVLOS) operations. All these applications need tools to accelerate and partially automate the creation of missions, the calculation of the optimal trajectories and the automatic execution of parts of the mission with the least human intervention, in order to obtain cost effective solutions. But also, quite often, at least critical parts of the flights need to be piloted by humans (e.g. due to the higher adaptability of human pilots to mission requirements).

Most of the aforementioned applications rely on the definition of ad-hoc procedures for missions' design, which tend not to consider the flight safety constraints, but assume a safe environment. It is clear it is not at all the case in urban environments, where there will be a large number of obstacles, areas restricted to flight, etc. And additionally, if the air traffic starts to grow, there will be needs to coordinate operations to avoid drone collisions and surely it will be necessary to impose physical constraints to flights (in the form of corridors, airways, and explicit rules of air, etc.), at least in some areas [17]. In order to ensure flight safety, a collection of concepts and tools been developed in the last few years, such as those related to the FAANASA UTM (Unmanned Traffic Management [18]) program, or to the European U-Space concept from SESAR program [19]. Also, there is quite a large amount of companies developing UTM solutions (most of the relevant actors are included in GUTMA association [20]), and the flight regulators (i.e. ICAO, AESA, etc.) are working in the problem of opening the sky for this new actors, with special focus in low level operations and in urban areas, where most of the business case resides. With respect to regulators, it is especially interesting the work of JARUS group [21], which is defining a methodology for risk assessment (called SORA [22]) with the final aim to be able to automate flight authorization, as part of the UTM process.

In this paper we will define a potential set of flight planning use cases, interfaces and requirements integrating the existing flight planning processes with UTM processes. Also, some hints on a UTM-integrated working prototype of this kind of system we are building will be provided. Summarizing, this flight planning tool needs to define flight plans for different applications to: 1) Cover different use cases and applications; 2) Enable the prediction of the risk of a given operation, using a SORA inspired methodology; 3) Coordinate the occupancy of the airspace, minimizing the chance of mid-air collisions.

The paper continues with a section devoted to a high-level description of some current example flight planning tools (section II), and then it focuses of UTM functional architecture (section III). Section IV describes the Flight Planning Use Cases, while Section V is devoted to the associated input and output data requirements. Then, Section VI describes the 
prototype flight planning tool we have implemented, and section VII includes some practical results. Finally, section VIII contains some conclusions and potential future research lines.

\section{Description of CURREnt Fligth Planning Systems}

There are currently in the market many tools for the definition of missions for drones, such as the ones developed by drone manufacturers (i.e. Parrot or DJI tools [23], [24]). Drone manufacturers tools, integrated with the drone control systems, usually follow similar concepts:1) They allow the user to flight manually the drone or to establish a set of waypoints that conform a path to be followed by the drone; 2) They rely on standard map technologies such as Google Maps and offer a 2D point of view; 3) They allow to create a flight plan and to automatically upload it to a drone for an automatic flight. For instance, in the case of Parrot, they use MAVLink [25], [26]

These tools are designed to enable a fast and easy way for the users to interact with them, to the detriment of more complex scenarios and systematic mission definition. Other companies provide more advanced platforms, specially devoted to specific applications and with associated tools for mission specification. Three examples of such platforms can be found in [27], [28], [29]. Also, for other drones such as those based on Pixhawk or Ardupilot autopilots, other similar tools as APM Planner 2 [30], with similar features and interfaces to the drones are available.

A more complex application-oriented solution is described in [6], where a system for partially automated design of infrastructure inspection is described, which, from 3D primitives, derives flyable flight plans and even translates them to a MAVLink specification. The academic ambit has also focused on the goal of generalizing the definition of the missions [31] where the authors developed a Domain Specific Language that enables setting mission specifications and predicting trajectories, similar to efforts in commercial aircraft trajectory predictions for Air Traffic Management [32]. In [33] it is also described the flight plan definition problem as a concatenation of legs joining waypoints, and [34] describes a complete language hierarchy and associated calculation engines, mainly devoted to the lower levels of the trajectory prediction problem for commercial aircraft and also for multi-rotors.

Other support tools to make possible for non-expert users (e.g. firefighters, rescue workers, etc.) to specify missions are also being delivered [35] [36]. Meanwhile, current commercial systems for UTM (i.e. Airmap [37] or Unifly [38]) allow for declaration of basically two types of flights: 1) Waypoint based flight plans;2) Flight Areas.

We find this kind of approaches especially appealing, as they allow for non-experts to be able to declare flight plans for their applications, irrespective of the actual type of mission. From the previous description of tools, and considering the main use cases, we will derive in section IV the requirements for a realistic Drone Flight planning tool for urban operations. But before, we need some additional background on the main functions of UTM and their relation.

\section{UTM FUNCTIONAL ARCHITECTURE DESCRIPTION}

The definition of the services which should be provided to users within UTM is currently under discussion, with different potential architectures. In all of them there will be a collection of services provided to the airspace users (drone operators and pilots) by either a central entity (i.e. an Air Navigation Service Provider, ANSP), or by a collection of UTM Service Providers (USPs), working collaboratively. A good summary of the intended services to be provided is detailed, taken (and summarized) from SESAR2020 U-Space documentation [39]. The table also shows the associated target time for services launch, in four phases called U1, U2, U3 and U4.

TABLE I. U-SPACE SERVICES

\begin{tabular}{|c|c|}
\hline $\begin{array}{c}\mathrm{U} 1 \\
(2019)\end{array}$ & $\begin{array}{l}\text { Registration, identification, provision of static } \\
\text { geofences (No drone zones, ...) to users }\end{array}$ \\
\hline $\begin{array}{c}\mathrm{U} 2 \\
(2022)\end{array}$ & $\begin{array}{l}\text { Drone Tracking, air safety monitoring (no } \\
\text { incursion in geofences, terrain avoidance, } \\
\text { conflicts between aircraft, ...), flight plan } \\
\text { authorization (including strategical deconfliction), } \\
\text { flight plan conformance monitoring, weather } \\
\text { provision to operators, access to aeronautic and } \\
\text { ATC data, emergency management, provision to } \\
\text { pilots of nearby traffic information }\end{array}$ \\
\hline $\begin{array}{c}\text { U3 } \\
(2027)\end{array}$ & $\begin{array}{l}\text { Dynamic (on board) geofencing, improved } \\
\text { ATC/ATM interface, tactical conflict detection } \\
\text { and resolution and drone congestion management }\end{array}$ \\
\hline $\begin{array}{c}\mathrm{U} 4 \\
(2035)\end{array}$ & $\begin{array}{l}\text { Full integration with ATC/ATM and manned } \\
\text { aviation and additional services }\end{array}$ \\
\hline
\end{tabular}

From the previous enumeration of services and considering the planning procedures described in the previous section it is clear the planning cannot be performed, in the near future, independently of the UTM systems. It is necessary that the flight planning systems and the UTM systems enable a partiallyautomated collaborative process to plan, authorize, and monitor flights, able to respond to potential contingencies. In Fig. 1., the main functions related to pre-flight processes are depicted, along with their main relations. Note here we are being agnostic with respect to the actual system architecture (centralized by an ANSP, provided by a federation of USPs, a mixture of both, ...). The central processes here are those related to Flight planning (Flight Planning Support and Flight Plan Translation in the figure), and those related to partially automated Flight authorization (Flight Plan assessment, Alternative Flight plan Assessment and Flight Authorization in the figure). All those functions need to take into account contextual information (both static and dynamic), potential manned traffic in the area, use accurate drone flight modelling, must and respect the drone operator's privacy concerns. Next, we will summarize each of the central pre-flight functions (the surrounding processes are named where the associated functionality is needed):

- Flight planning support should provide means to easily define most typical flight plans, though an attached map based (potentially 3D) HMI. The definition of such a flight plan is the main focus of the paper. 
- Flight Plan Translation is another process enabling the use of most specialized flight planning tools by the operators, by performing a translation of those flight plans to a format which may be understood by the authorization processes.

- Flight Authorization process uses the flight plan specification, and by calling the Flight Plan Assessment process decides if the flight might be authorized or not (or in certain cases, may request for human assessment). If the flight plan is not authorized, it may also automatically provide amended flight plans to the flight planning support service (designed by the Alternative Flight Plan proposal process), so that the operator may establish an informed negotiation process (but without having access to other operators' flight plans). Also, this process might ban a previously authorized flight due to changes of the Dynamic Flight Context (i.e. new geofences, sudden change in predicted weather, etc.).

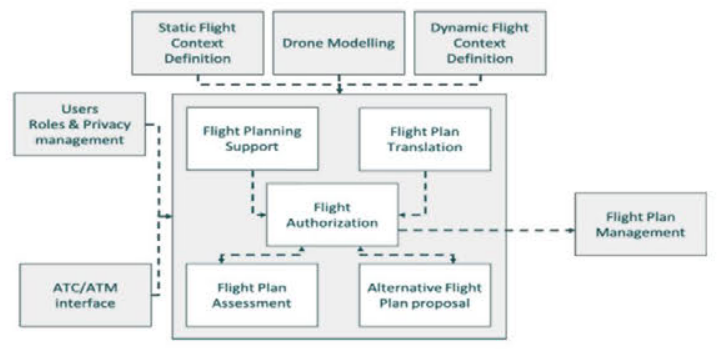

Fig. 1. Flight Planning and Authorization.

- Flight Plan Assessment Process must consider Static Flight Context (air rules, static geofences, terrain, obstacles), Dynamic Flight Context (Weather, dynamic geofences, communication and navigation systems availability and integrity, ground occupancy by people, urban traffic, etc.) and previously authorized flights to assess the safety of each flight. To do so, it needs an accurate space-time description of the flight, which at the same time needs to be operationally consistent with drone operator mission needs.

- Alternative Flight Plan proposal process must be able to amend requested but not authorizable flight plans, providing alternatives which are authorizable while respecting (as much as possible) mission definition. From this definition, it is clear the drone operators would need to specify parts of their operations to be critical (no mission would be performed if this is not respected) and others which may be changed by this process, if needed (i.e. altitudes while performing an approach to the place where a delivery is to be performed, or times to start a not-time critical mission).

Once a flight plan gets authorized, it would enter the Flight Plan Management process, which is the link between the preflight and the along-flight services within the UTM. The processes associated to along-flight services are depicted in Fig. 2. In this case we basically the UTM system tracks all drones and continuously check these flights follows the authorized flight plans, do not invade any geofence or problematic area, do not become too close (to avoid mid-air incidents or accidents) and there are no associated contingency problems such as loss of command \& control communications or loss of navigation. Next, we will summarize the central along-flight functions:
- Flight Plan Management is in charge of maintaining the current state of the flight: is it active (the drone is flying), terminated, did the plan got revoked?

- Air Monitoring function does all the safety assurance functions related to each individual aircraft: Drone Tracking, incursion in geofences monitoring, terrain avoidance monitoring, flight plan conformance monitoring, and provision to pilots of nearby traffic information (including both manned and unmanned aircraft). To do this, it must consider the current Static and Dynamic Flight context.

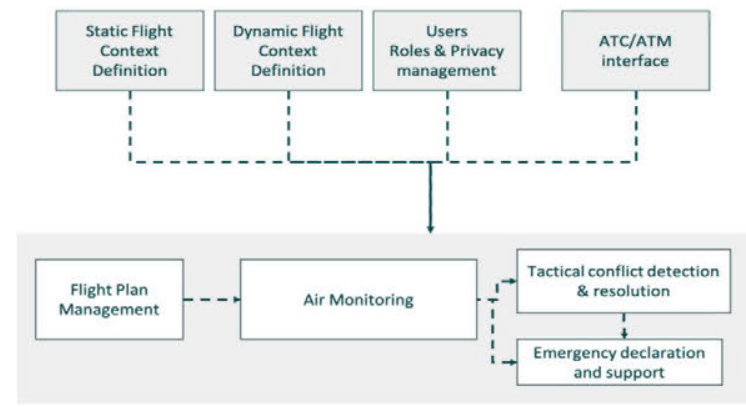

Fig. 2. Flight Monitoring and Safety Assurance.

- Meanwhile, Tactical Conflict Detection and Resolution Process checks potential near future loss of separation for drones, according to their tracks and to their intended flight plans.

- Finally, due to the potential safety events occurring during the flight, an emergency declaration and support to resolution process will need to assess no major hazard arises, and, if necessary, provide the involved authorities with tools to manage the event (i.e. means to automatically and safely terminate flights, backup solutions, etc.).

Once we stated UTM functions, at a very high level, we will start describing more in detail flight plan needs, flight plan data requirements and flight planning tools.

\section{FLIGHT PLANNING SYSTEMS USE CASES}

Although in current civilian drone operations most of the systems are focused on performing VLOS operations, as described in section II, it is clear that many of the future applications of drone technologies to urban areas will demand implementation of safe BVLOS flights. Coincidentally, the near-future deployment of $5 \mathrm{G}$ networks has the potential to allow for safe command and control and information retrieval from drones. Therefore, this is the kind of environment (low latency and high bandwidth communications) we will assume for the description of the following use cases. We will also not make assumptions about the presence of intelligence on-board (enabling drone autonomy), but will assume the coordinated efforts from on-board and ground systems (including potentially human pilots at least for contingency management) allows to perform two types of operations:1) Automated flight, following waypoints. This kind of operation is central for translation of the drone from its take off-area to the actual area of business related operations and from there to the intended landing area, or for goods delivery; 2) Manual/autonomous flight, for high precision 
remote operations (i.e. focused inspection, precision landing, area surveillance).

Based on these ideas, we will next define the flight planning use cases and from them we will derive a more detailed description if the requirements of an integrated flight planning process for urban operations.

\section{A. Pre-flight Use Cases}

Next, we will describe several use cases for flight planning and authorization, describing the interactions between the different functions and the potential outcomes.

1. Waypoint based flight planning: In this use case, the operator needs to design a flight plan to make the drone move from a take-off location to a landing location. He will make use of the Flight planning support process, which provides him with up to date information about the airspace "airways" structure (so he fills only a few waypoints and automatically the system fills additional waypoints), geofences, weather situation, etc. All the flight plan is designed iteratively, and the flight plan support system calculates, based on drone dynamics, an interval for the expected time of arrival to every waypoint. Also, the operator must define a maximum and minimum initial time for take-off. The support system also checks the flight may be completed by the drone, given its endurance.

2. Manual/autonomous area operation flight planning: In this case, the mission is assumed to need a manual/autonomous flight section, where we are not able to establish waypoints before flight. Instead, the flight plan is defined through an area (in fact a 3D volume) to be reserved for a given time interval. The reason for this, quite often, is that depending on the information being retrieved from the drone, the actual flight operation may change (i.e. in emergency or police operations, in large area infrastructure inspection operations, ...), or the operator does not want to give too much details due to its business constraints. This kind of operations may also be used to model final landing in a not fully controlled environment. Also, this could be used to plan drone swarm operations without defining individual flight plans. In this case the flight plan is designed at once, and there is no point in trying to use drone dynamics to calculate time intervals. The operator must specify flight duration and a maximum and minimum initial time for take-off. Also, the operator needs access to up to date information about the airspace "airways" structures, geofences, weather situation, etc. to design its flight plan in a safe way.

3. Hybrid operations flight planning: Here we may want to plan, for instance, the consecutive delivery of several goods to different not fully controlled landing areas, or the inspection of several infrastructures in a single flight. In this case, the flight plan may be composed of a concatenation of waypoint lists and manual/autonomous flight areas. This case has the two previous ones as particular cases.

In any of the previous use cases, after designing the flight plan, the user provides it to the flight authorization process which either authorizes it or denies it. In the second case, it is possible the authorization system provides alternative flight plans which could be used by the operator to either send it back and get almost automatic approval, or as a basis for flight plan refinement on its side and the start of a new authorization process. To make the negotiation process faster, the operator might mark several parts of the flight as business-critical. In fact, there are cases where it is business-critical to perform the flight not only following a defined path or in a given area, but also conforming tight time constraints (i.e. when the drone is used to perform surveillance of a given event in a certain position and time).

The definition of time constraints for each part of the system is a hard requirement for different parts of the UTM system. Specifically, Flight Plan Assessment needs the time in order to be able to predict potential interactions between several drones, or to assess the impact of weather or the occupancy of the ground. Also, and especially for fixed-wing drones, it is necessary that the planning tools help the user to constrain flight heights to avoid obstacles, and as previously stated, to avoid forbidden areas and respect potential airways structures.

Finally, there are other two use cases, related to the potential revoking of a previously allowed flight plan by the UTM (due to changes in the Dynamic context), and to the removal of authorized flight plans by their creators. In the first case, the flight planning HMI seems the most adequate channel to inform both operators and drone pilots of this kind of event. In the second, the same HMI may be used to select an authorized flight plan and requests its removal.

Depending on the kind of operation, it is clear the capacity to rapidly fill in an authorizable flight plan, and all the associated data, will be of paramount importance from the business perspective. This would be the case of emergency services, fast delivery of critical goods (or freshly cooked food), etc.

\section{B. Along-flight Use Cases}

During flight Air Monitoring will make use of the flight plan to detect potentially hazardous events such as:

- Lack of conformance of actual flight with authorization both in space (getting out of approved area or too far away laterally or vertically from the lines connecting waypoints) or in time (not respecting the authorized time constraints).

- Future tactical conflicts (losses of separation) between drones in the near future, to be assessed making use their intent to enhance the dynamic predictions of current flights.

- Future interactions with dynamic geofences, with hazardous changing weather, etc.

Some of those events, when detected, will result in the need to perform a contingency procedure and abort the flight operation in a safe manner. But in some cases, there could be a procedure to allow for flight plan edition to resolve the situation, following a fast flight-planning-to-UTM negotiation process. This process, similar to that of the authorization, could result in the edition of business-critical parts of the flight. Again, in order to guarantee operations safety, it is not only necessary that the geometry of the flight is defined, but also flight planning system and UTM need to have a shared view of the time constraints of the flight (even tighter during actual flight operation). 


\section{FLIGHT PLANNING DATA REQUIREMENTS}

From the previous use cases, next we will derive a collection of data requirements for the specification of missions (input of the Flight Planning Support process), and for the provision of flight plans for the rest of the UTM system (out of the Flight Planning Support process)

\section{A. Input Mission specification data Requirements}

Next, we will detail the flight plan specification data fields, with some rationale:

- Identification data (for flight plan, operator, pilot, drone, ...). Necessary to check compliance to regulation, certification, and to define the adequate drone dynamics.

- Priority

- Drone flight endurance.

- Take off area: It is recognized in many cases it is impossible to define a take-off point at planification time.

- Take-off time interval: quite often the time needed preparation and deployment of the drone are not completely controllable, so it is necessary to open a time window.

- A collection of flight phases, which are either collections of successive waypoints (in 2D or 3D), with a potential groundspeed constraint, and a flag saying if this flight phase is business-critical (with respect to its spatial design or also considering times to be calculated by the system), and areas (volumes), defined through polygons, with associated minimum and maximum duration of the flight within them. Again, there should be a flag indicating if this flight phase is business-critical.

- A landing area: again, it is acknowledged in many cases it is impossible to define a landing point at planification time.

\section{B. Output Flight Plan specification data Requirements}

From the previous data, the flight planning support process must complete a flight plan containing, in addition to previous data, the following fields, demanded by some parts of the UTM system either at pre-flight or along-flight (with rationales):

- A collection of flight phases. The output flight plan may not only have the input waypoints, but some additional ones due to the need to be compatible with the airspace structures (such as airways or corridors), or to avoid certain obstacles present in direct paths. In addition to previous (input) fields, the flight plan needs to contain time and altitude constraints for all way points, calculated considering drone dynamics, to enable proper safety assessment and alternative flight proposals, and also to allow flight plan conformance monitoring in the vertical and time dimensions.

- A landing time interval calculated using drone dynamics.

\section{Prototy Pe Flight Planning ToOL}

In this section we will describe, at a very high level, a flight planning tool prototype partially implementing the aforementioned use cases.

\section{A. Service Architecture and contextual data}

Our flight planning tool is built making use of a microservice architecture, connecting contextual data retrieved from outer and internal Web Services, and based on the extensive use of REST APIs for service-oriented communications and MQTT for notification publication of asynchronous events. The front-end is built in HTML5/CSS/JavaScript, and the central parts of the backend logic are built either in JavaScript (Node.js) or making use of $\mathrm{C}++$ for computationally complex parts of the system (specifically, for trajectory prediction). For trajectory prediction we are making use of the same kind of techniques outlined in [6], which are an extension of the methods described in [32].

The system connects to the following services to obtain upto-date data to describe both static and dynamic flight context:

- Mapping information is retrieved from Google Maps ${ }^{\circledR}$, which is also used as the basis for the map-based interface.

- Digital terrain (elevation and surface) models are retrieved from the Spanish National Geographic Institute (IGN [40]).

- Weather data is retrieved from public servers such as Open weather [41].

- $3 \mathrm{G} / 4 \mathrm{G}$ communications services or navigation services performance are not assessed or used in our prototype.

- Static Geofencing is automatically created on the basis of a digital version of Spanish AIP, and other public databases (i.e. hospitals, nuclear facilities, national parks, etc.).

- Dynamic Geofencing is retrieved from the a full-scale UTM prototype we are also developing, with interfaces for state services (police, firefighters, etc.).

It makes use of the aforementioned information to either perform trajectory predictions (mapping, digital terrain, and wind data are used at this stage), or to provide visual information to the flight plan designer, so he creates flights compatible with the current flight context (for the time of the operation).

\section{B. Example HMI and operations}

Fig. 3 is a representation of a potential 2D map-based visualization HMI for such a tool. Here you may see the map, a collection of waypoints over the map, with an associated sequence number. After providing as inputs all the data described in section V.A (by map clicking or textual information providing), the tool would calculate the output described in section V.B.

The resulting flight representation is given in Fig. 4. There, it is especially relevant the capability to see the time/altitude evolution of the flight vs the traveled distance. In this representation it is readily visible at the bottom-left of the figure (also expected flight landing time interval is provided there). 


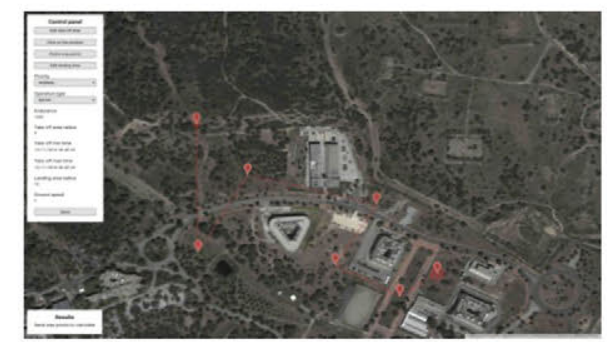

Fig. 3. Flight Data Input.

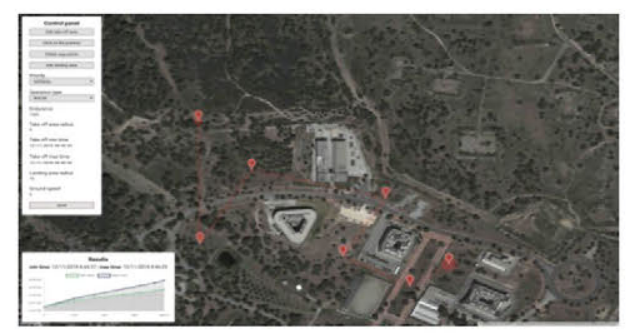

Fig. 4. Flight Data Output.

\section{EXAMPLE FLIGHT PLANNING}

In this section we will exemplify a complete flight planning exercise. Let us assume we need to plan a delivery of one critical item from one of our university premises to another one. We have to do so crossing a heavy traffic area, where a set of crossing airways, with several heights, has been implemented to organize traffic and avoid unsafe operation over crowds. The take-off area, landing area, airways infrastructure (green lines) and geofences (red areas) are depicted in Fig. 5.

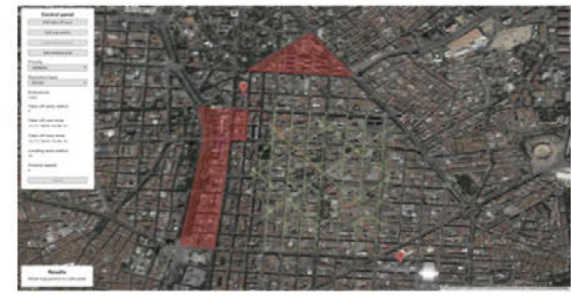

Fig. 5. Take-off and Landing Areas and airspace structure.

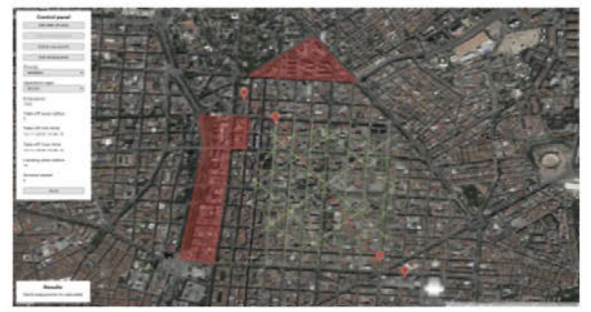

Fig. 6. Flight Plan Input.

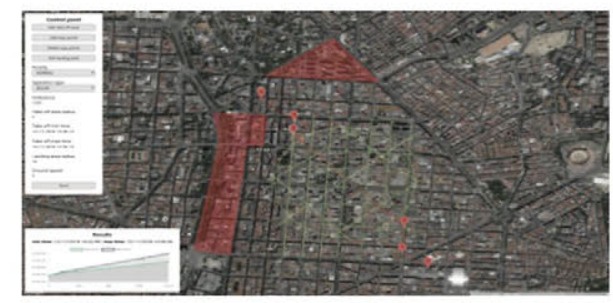

Fig. 7. Flight Plan to be passed to request authorization.
In Fig. 6 we depict a hand-made flight plan, provided as input to the flight planning tool. Meanwhile, Fig. 7 represents the output of the flight plan support system, which in this case slightly edits the flight so it follows the airways infrastructure. Of course, this flight plan will still need to go through a UTM authorization process.

\section{CONCLUSIONS AND FURTHER WORK}

This paper describes a collection of typical urban drone mission types and establishes the interfaces between flight planning systems and UTM systems. Its basic contribution is the analysis of the need to integrate the planning phases in the whole traffic management solutions, to enable fast design of safe flight plans. Associated use cases and data requirements have been derived. The described processes are being implemented in a full-scale UTM prototype to enable experimentation of most advanced UTM concepts, such as tactical and strategical deconfliction of traffic, capacity management, etc.

\section{ACKNOWLEDGMENT}

This work was supported by UPM Project "Tecnologías Avanzadas para la Monitorización y Gestión Remota del Tráfico Aéreo de Vehículos Pilotados y no Pilotados" (RP1509550C02), and by the Spanish Ministry of Economy and Competitiveness, Grant TEC2017-88048-C2-1-R.

\section{REFERENCES}

[1] SESAR Undertaking, "European Drones Outlook StudyUnlocking the value for Europe", SESAR, Brussels, 2016.

[2] M. \&. A. A. Hassanalian, "Classifications, applications, and design challenges of drones: A review", Progress in Aerospace Sciences, 2017.

[3] Amazon. Amazon Prime Air, 2016. Available online at https://www.amazon.com/Amazon-PrimeAir/b?node $=8037720011$.

[4] Stimpson, A., Cummings, M., Nneji, V. C., \& Goodrich, K. H. (2017). "Exploring Concepts of Operations for OnDemand Passenger Air Transportation".

[5] Ariff, O. K. (2017). "Introduction to Unmanned Aircraft Systems", Second edition DM Marshall et al CRC Press. The Aeronautical Journal, 121(1244), 1592-1595.

[6] Besada, J. A., Bergesio, L., Campaña, I., VaqueroMelchor, D., López-Araquistain, J., Bernardos, A. M., \& Casar, J. R. (2018). "Drone Mission Definition and Implementation for Automated Infrastructure Inspection Using Airborne Sensors". Sensors, 18(4), 1170.

[7] Richman, B., Bauer, M. P., Michini, B. J., \& Poole, A. J. (2017). U.S. Patent No. 9,609,288. Washington, DC: U.S. Patent and Trademark Office.

[8] Barkham, R., Bokhari, S., \& Saiz, A. (2018). "Urban Big Data: City Management and Real Estate Markets". GovLab Digest: New York, NY, USA.

[9] de Melo, R. R. S., Costa, D. B., Álvares, J. S., \& Irizarry, J. (2017). "Applicability of unmanned aerial system (UAS) for safety inspection on construction sites". Safety science, 98, 174-185. 
[10] Rossi, M., Brunelli, D., Adami, A., Lorenzelli, L., Menna, F., \& Remondino, F. (2014, November). "Gas-drone: Portable gas sensing system on UAVs for gas leakage localization". SENSORS, 2014 IEEE (pp. 1431-1434). IEEE.

[11]Rau, J. Y., Jhan, J. P., Lo, C. F., \& Lin, Y. S. (2011). "Landslide mapping using imagery acquired by a fixedwing UAV'. Int. Arch. Photogramm. Remote Sens. Spat. Inf. Sci, 38, 1-C22.

[12] Niethammer, U., Rothmund, S., Schwaderer, U., Zeman, J., \& Joswig, M. (2011). "Open source image-processing tools for low-cost UAV-based landslide investigations". International Archives of the Photogrammetry, Remote Sensing and Spatial Information Sciences, 38(1), C22.

[13] Carvajal, F., Agüera, F., \& Pérez, M. (2011, September). "Surveying a landslide in a road embankment using unmanned aerial vehicle photogrammetry". International Conference on Unmanned Aerial Vehicle in Geomatics (UAV-g) (pp. 14-16).

[14]Branco, L. H. C., \& Segantine, P. C. L. (2015). "MaNIACUAV-a methodology for automatic pavement defects detection using images obtained by Unmanned Aerial Vehicles". Journal of Physics: Conference Series (Vol. 633, No. 1, p. 012122).

[15]Máthé, K., \& Buşoniu, L. (2015). "Vision and control for UAVs: A survey of general methods and of inexpensive platforms for infrastructure inspection". Sensors, 15(7), 14887-14916.

[16] González-Jorge, H., Martínez-Sánchez, J., \& Bueno, M. (2017). "Unmanned aerial systems for civil applications: A review". Drones, l(1), 2.

[17] Airbus. Premiering a future blueprint for our sky, 2018. Available online at: https://www.airbus.com/innovation/Premiering-a-futureblueprint-for-our-sky.html.

[18]NASA. FAA-NASA UTM, 2015. Available online at: https://utm.arc.nasa.gov/index.shtml.

[19] SESAR, 2014. Available online at: https://ec.europa.eu/transport/modes/air/sesar en

[20] Global UTM Association. Available online at: https://gutma.org/.

[21] JARUS: Joint Authorities for Rulemaking on Unmanned Systems. Available online at: http://jarus-rpas.org/.

[22] SORA: Specific Operations Risk Assessment (SORA), JARUS, 2017. Available online at: http://jarusrpas.org/content/jar-doc-06-sora-package.

[23]Bebop drone. Standard, Parrot. Available online at: http://developer.parrot.com/docs/bebop/.

[24] Dà-Jiang Innovations Science and Technology Co., Ltd. DJI, 2017. Available online at: https://www.dji.com.

[25]Dietrich, T., Andryeyev, O., Zimmermann, A., \& Mitschele-Thiel, A. (2016, May). "Towards a unified decentralized swarm management and maintenance coordination based on mavlink". Autonomous Robot Systems and Competitions (ICARSC), 2016 International Conference on (pp. 124-129). IEEE.

[26] Atoev, S., Kwon, K. R., Lee, S. H., \& Moon, K. S. (2017, November). "Data analysis of the MAVLink communication protocol". Information Science and Communications Technologies (ICISCT), 2017 International Conference on (pp. 1-3). IEEE.

[27] DroneDeploy. DroneDeploy, 2017. Available online at: https://www.dronedeploy.com.

[28]DroneUp. DroneUp, 2017. Available online at: https://www.droneup.com.

[29]Drone Base, Inc. Drone Base, 2017. Available online at: https://www.dronebase.com.

[30] APM Planner 2. Ardupilot, 2016. Available online at: http://ardupilot.org/planner2/.

[31] Ruscio, D. D., Malavolta, I., Pelliccione, P., \& Tivoli, M. (2016, October). "Automatic generation of detailed flight plans from high-level mission descriptions". Proceedings of the ACM/IEEE 19th International Conference on Model Driven Engineering Languages and Systems (pp. 45-55). ACM.

[32]Frontera, G., Besada, J. A., Bernardos, A. M., Casado, E., \& López-Leonés, J. (2014). "Formal intent-based trajectory description languages". IEEE Transactions on Intelligent Transportation Systems, 15(4), 1550-1566.

[33]Pastor, E., Santamaria, E., Royo, P., Lopez, J., \& Barrado, C. (2010, March). "On the design of a UAS flight plan monitoring and edition system". Aerospace Conference, 2010 IEEE (pp. 1-20). IEEE.

[34]Frontera Sánchez , G. (2016) "Applications of Formal Languages to Management of Manned and Unmanned Aircraft". PhD thesis, Universidad Politécnica de Madrid, Signal, Systems and Radiocommunications Department, Madrid, Spain, 2016. Available online at: http://oa.upm.es/40622/.

[35] http://www.drones-112.com

[36] Bozhinoski, D., Di Ruscio, D., Malavolta, I., Pelliccione, P., \& Tivoli, M. (2015, November). "Flyaq: Enabling nonexpert users to specify and generate missions of autonomous multicopters". Automated Software Engineering (ASE), 2015 30th IEEE/ACM International Conference on (pp. 801-806). IEEE.

[37] Airmap. Airmap. Available online at: https://www.airmap.com/.

[38] Unifly. Unifly (2018). Availble online at: https://www.unifly.aero/.

[39] U-Space. SESAR (2018). Available online at: https://www.sesarju.eu/U-space.

[40] Spanish National Geographic Institute. Available online at: http://www.ign.es/web/ign/portal.

[41] OpenWeatherMap. Available online at: https://openweathermap.org/. 